\title{
FPGA REALIZATION OF SOBEL EDGE DETECTION ALGORITHM FOR BREAST CANCER DETECTION USING THERMAL IMAGES
}

D. Selvathi

Senior Professor, Department of Electronics and Communication Engineering, MEPCO Schlenk Engineering College Sivakasi, (India). E-mail: dselvathi@mepcoeng:ac.in ORCID: https://orcid.org/0000-0003-1159-3879

S. Bama

Associate Professor, Department of Electronics and Communication Engineering, Kalasalingam Academy of Research and Education, (India).

E-mail: bamasrini@yahoo.com ORCID: https://orcid.org/0000-0002-9450-0692

\section{Gitación sugerida:}

Selvathi, D., y Bama, S. (2021). FPGA Realization of sobel edge detection algorithm for breast cancer detection using thermal images. 3C Tecnología. Glosas de innovación aplicadas a la pyme, Edición Especial, (noviembre, 2021), 443-457. https://doi.org/10.17993/3ctecno.2021.specialissue8.443-457 


\section{ABSTRACT}

According to World Health Organization, breast cancer is the most prevailing cancer among women which claims thousands of lives each year. Mammogram imaging modality is the popular traditional diagnosing tool used for breast cancer screening. However, one in 5 breast cancers have been missed in this screening as these machines are incapable of detecting it in early stage. Hence emerging thermography procedure is also suggested for clinical records. It produces the skin surface temperature as a thermal pattern imaging. The aim of this work is to detect breast cancer using thermographic images. Thermal images available in the DMR database have been employed for this analysis. The color conversion using $\mathrm{YcRcB}$ color model is carried out to extract the features from raw image. Gray tone images are obtained using thresholding. Sobel edge detection algorithm is used to segment normal and abnormal images. The image preprocessing and thresholding are done in MATLAB and segmentation algorithm is implemented in SPARTAN-3 Tyro plus FPGA kit using EDK. This developed system may help the doctors to give a second opinion.

\section{KEYWORDS}

Breast cancer, Thermal Images, FPGA, Segmentation. 


\section{INTRODUCTION}

Breast cancer in women is the second most occurring cancer because of abnormal growth of normal cells. International Agency for Research on Cancer (IARC) says that approximately one among eight ladies is plagued by cancer in life time and over 4,00,000 women are suffering from cancer and is increasing as a result of detecting cancer in its advanced (American Cancer Society, 2014). It justifies the need of research for early diagnosis to reduce mortality rate, avoid of surgical and increases survival rate. Manual diagnosis is tedious and time-consuming process (Kukar, 2003). And also, it possesses high degree of intra and inter observer variation (Eddy, 1990; Lilford et al., 1998). There is a need of computer aided diagnosis system with expert intelligence which will assist the physicians to eliminate the difficulties in diagnosis (Rodrigues et al., 2014; Übeyli \& Güler, 2005a). Several techniques are developed for automated diagnostic systems to attempt to solve this problem. Such techniques work for transforming qualitative diagnostic criteria into a more objective quantitative feature classification problem (Kordylewski, Graupe, \& Liu, 2001; Kwak \& Choi, 2002; Übeyli \& Güler, 2005b).

Mammography is widely used in clinical applications and mass screening due to its inexpensiveness, less time consumption and low complexity but fails to detect abnormality at an early stage because mammogram looks at structural or anatomical parts of the body. To overcome this disadvantage, diagnosis is done based on metabolic activity, the blood flow and the temperature on the surface of the skin. A special heat sensing camera is used to measure and map heat on the surface of breast. The skin temperature reflects abnormality in the tissue such as the presence of a tumor which is used as indicators of a disease.

Breast Thermography is widely used to diagnose the breast cancer. It is a relatively new screening technique based on temperature sensing. Breast thermography as a diagnostic tool for tumor detection is based on the fact that cancerous and precancerous tissues have high metabolic rate. As a consequence, the temperature of that area is higher compared to normal breast tissues temperature, thus tumors that are small in size can be well identified using thermography. This technique is entirely non-invasive, fast and painless as it requires no contact between the patient and instrumentation (Milosevic, Jankovic, \& Peulic, 2014). 
Infrared thermography has been proven to be a promising technique on early diagnosis of breast pathologies (Ankit \& Mandal, 2015). Field Programmable Gate Arrays (FPGA) is a high-speed reprogrammable processor that can store several pieces of software to do different tasks and entirely change its hardware in a matter of milliseconds. The re-programmability of FPGAs allows them to be used to implement any architecture. "Spartan 3E Tyro Plus" is a FPGA board produced by Xilinx (XILINX, n.d.). Many works are done for Breast Cancer Diagnosis (Li et al., 2011; Niwas et al., 2013). The skin tone segmentation and classification are performed and implemented in FPGA (Niwas et al., 2013). The K means clustering is used for color-based segmentation of hot region for breast cancer detection using thermograph (Gayathri, Madhavi, \& Bobby, 2015; Hankare et al., 2016; Nausheen et al., 2018). In this work, Edge based segmentation with preprocessing techniques are done using FPGA implementation.

\section{MATERIALS AND METHODS}

The methodology used for FPGA implementation of breast cancer detection using thermal images is shown in Figure 1. The breast cancer images are obtained from the Database Mastology Research (DMR) database. The Color conversion is done using the color models like HSV, YIQ and YcRcB in MATLAB and the best model with high accuracy is selected. The segmentation is done initially by thresholding using the histogram and further segmentation is done using the Sobel edge detection algorithm. The thresholding using histogram is implemented in MATLAB. The Sobel edge detection algorithms are implemented in FPGA.

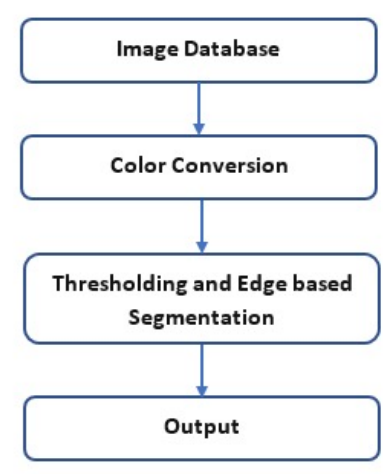

Figure 1. Flow diagram of breast cancer detection using thermal medical images.

Source: own elaboration. 


\subsection{DMR DATABASE}

DMR is an online platform that stores and manages mastological images for early detection of breast cancer. The database consists of 258 records of thermal images shot by thermal camera taken from different patients.

\subsection{COLOUR CONVERSION AND THRESHOLDING}

The color analysis is mainly done to separate Red, Green and Blue colors from the base image and then color models like $\mathrm{HSV}, \mathrm{ycRcB}$, etc. are carried out to extract the features and for further study and analysis of the image. The image is obtained from the database and the color conversion and color models are applied on it.

The $\mathrm{ycRcB}$ color model is used for the color conversion, since the base image is mainly composed of green, yellow, red and orange. An efficient output of feature is obtained from the color model where $\mathrm{cR}$ - Red chroma, cB - Blue chroma, Y - Luminance. In this, Luminance mainly represents the Gray scale Information, Red chroma and Blue chroma components carry color information.

$$
\begin{aligned}
& Y=0.299 * R+0.587 * G+0.1114 * B \\
& C_{R}=0.17327 *\left(R-Y^{\prime} 601\right) \\
& C_{B}=0.56433 *\left(B-Y^{\prime} 601\right)
\end{aligned}
$$

Thresholding is mainly done to separate the obtained feature based on the black scale and white scale separation. Based on the contrast in the grayscale region (ie), gray scale (white) and high gray scale (black), the extraction is done. The equation 4 represents the formula for the histogram-based thresholding.

$$
b(x, y)=\left\{\begin{array}{c}
1 \text { if } f(x)>T \\
0 \text { Otherwise }
\end{array}\right.
$$

The image pre-processing like color conversion and thresholding-based segmentation are done in MATLAB. 


\subsection{EDGE DETECTION ALGORITHM}

The Sobel Edge detection algorithm is used for the segmentation. Since edges occur at image locations to represent object boundaries, edge detection is extensively used in image segmentation. Representing an image by its edges has the further advantage that the amount of data is reduced significantly while retaining most of the image information. Since edges consist of mainly high frequencies, the edges can be detected by convolving the image with an appropriate kernel in the spatial domain.

\section{HARDWARE IMPLEMENTATION}

The block diagram for hardware implementation is shown Figure 2. The image is resized and a processor is created in FPGA using EDK to implement the image processing segmentation algorithm.

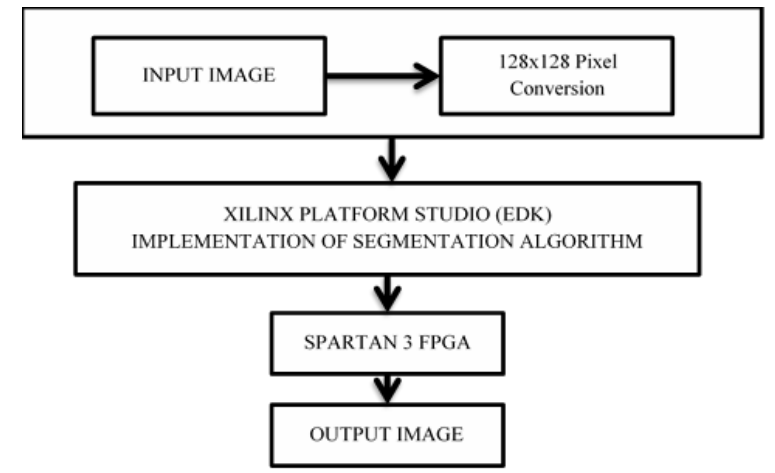

Figure 2. Block diagram for Hardware Implementation.

Source: own elaboration.

Initially the image is converted into $256 \times 256$ matrix and further it is converted into text file. This file is used in the coding for the execution. Figure 3 shows the architecture for the implementation of Sobel algorithm in FPGA. The equation 5 and 6 is used to obtain the Gradient Gx and Gy and equation7 is used to obtain the edge. The edge is calculated from the absolute values of the gradients.

$$
\begin{aligned}
& G_{x}=\left(P_{2}+P_{0}\right)+\left(\left(P_{5}-P_{3}\right)<1\right)+\left(P_{8}+P_{6}\right) \\
& G_{y}=\left(P_{6}+P_{0}\right)+\left(\left(P_{1}-P_{7}\right)<<1\right)+\left(P_{8}+P_{2}\right) \\
& S_{\text {out }}=\min \left(a b s\left(G_{x}\right)+a b s\left(G_{y}\right)\right), 255
\end{aligned}
$$




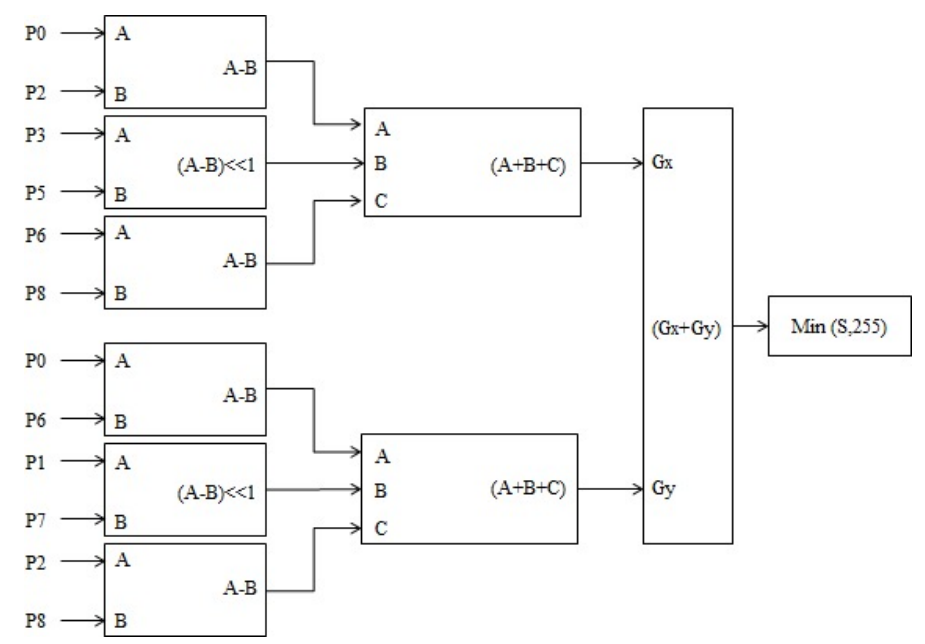

Figure 3. Architecture for Sobel Algorithm.

Source: own elaboration.

\section{RESULTS}

The database consists of both cancerous and non-cancerous images. The images are subjected to pre-processing and thresholding to extract features. Classifications are carried out in the XILINX PLATFORM STUDIO in FPGA SPARTAN-3 TYRO PLUS using EDK. For giving the input, the images pixels are converted to $1 \mathrm{D}$ and stored in the SRAM.

The proposed method is implemented using VHDL code by the structural modeling system. ISIM simulator tool in the XILINX project navigator 14.6 is used for simulation and the hardware implementation is done in FPGA using EDK in XILINX 10.1

\subsection{SIMULATION RESULTS}

Figure 4 shows the raw thermal image samples that are taken from the DMR image database. Sample 1 is normal image, sample 2 is cancer affected images. Figure 5 and 6 shows the color conversion of the base image taken from the database. The Luminance, Red chroma and Blue chroma is obtained separately. 


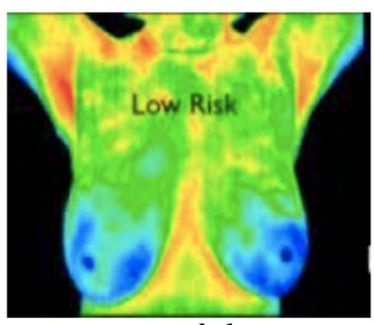

sample 1

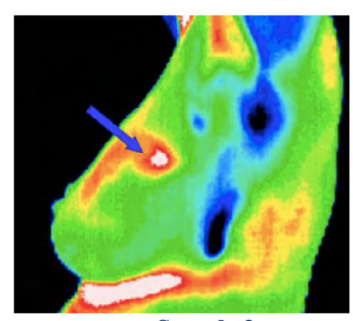

Sample 2

Figure 4. Raw Thermal image sample taken from DMR database.

Source: own elaboration.

\subsection{SEGMENTATION}

a) Thresholding:

The thresholding is done on the y component of the $\mathrm{ycR}_{\mathrm{cB}} \mathrm{B}$ image. Histogram is a graphical representation of pixels of image based on gray scale separation. The pixels of y component image are mapped in graph based on intensity. A mean value is taken to separate the low gray scale and high gray scale. The vertical lines in the graph represent the pixel values mapping based on intensity. The higher peaks represent the places where the intensity is very high and smaller peaks represents where the intensity of pixel is less. The low gray scale separation, high gray separation and pixel separation graph of different images are shown in Figure 7 and 8.

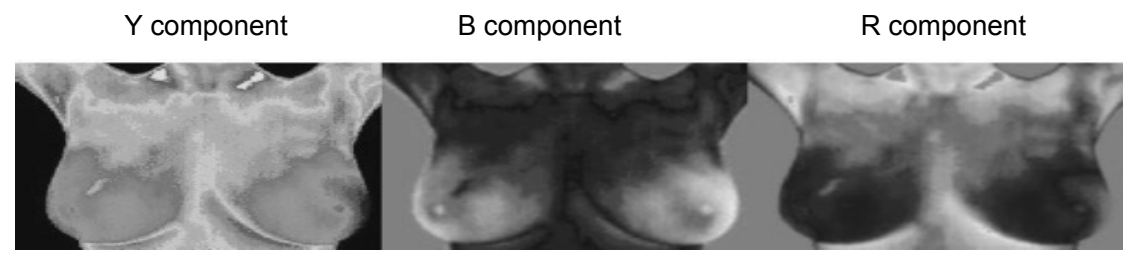

Figure 5. Flow YcRcB (Sample 1).

Source: own elaboration.

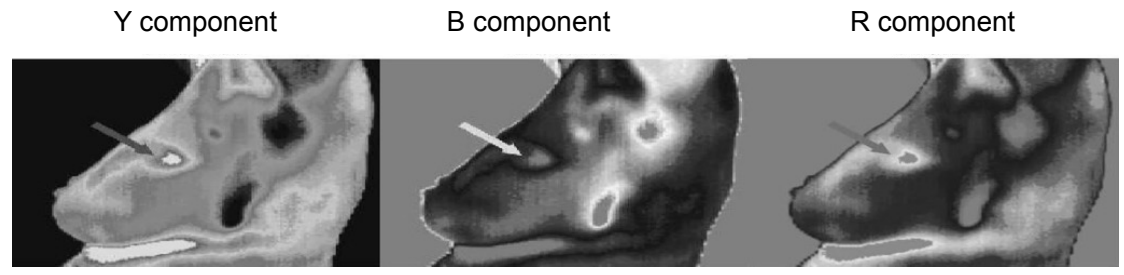

Figure 6. YcRcB (Sample 2).

Source: own elaboration. 
Low GrayScale

High GrayScale

Pixel Separation Graph

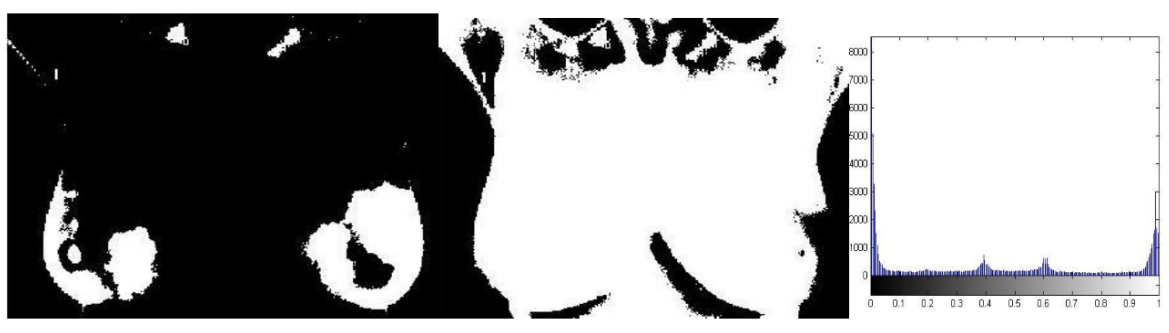

Figure 7. Thresholding (Sample 1).

Source: own elaboration.

Low GrayScale

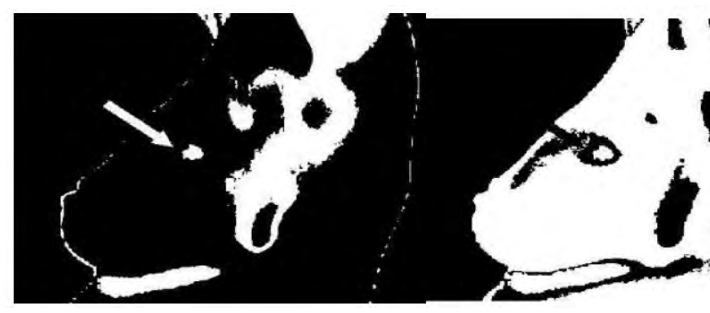

High GrayScale

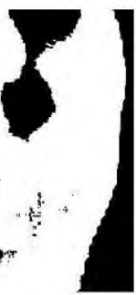

Pixel Separation Graph

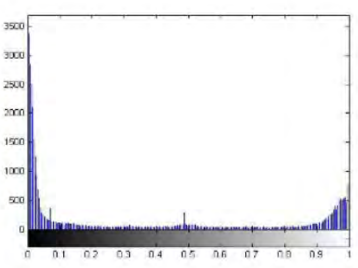

Figure 8. Flow Thresholding (Sample 2).

Source: own elaboration.

b) Edge Detection Algorithm Result in Verilog:

The 256×256 image pixel values are initially stored as 8 bit data in an SRAM. Each 8 bit number represents each pixel value of the 256x256 image. Figure 9 shows the output waveform of pixel storage in SRAM. After pixels stored in the SRAM, the Sobel edge detection algorithm is executed. 


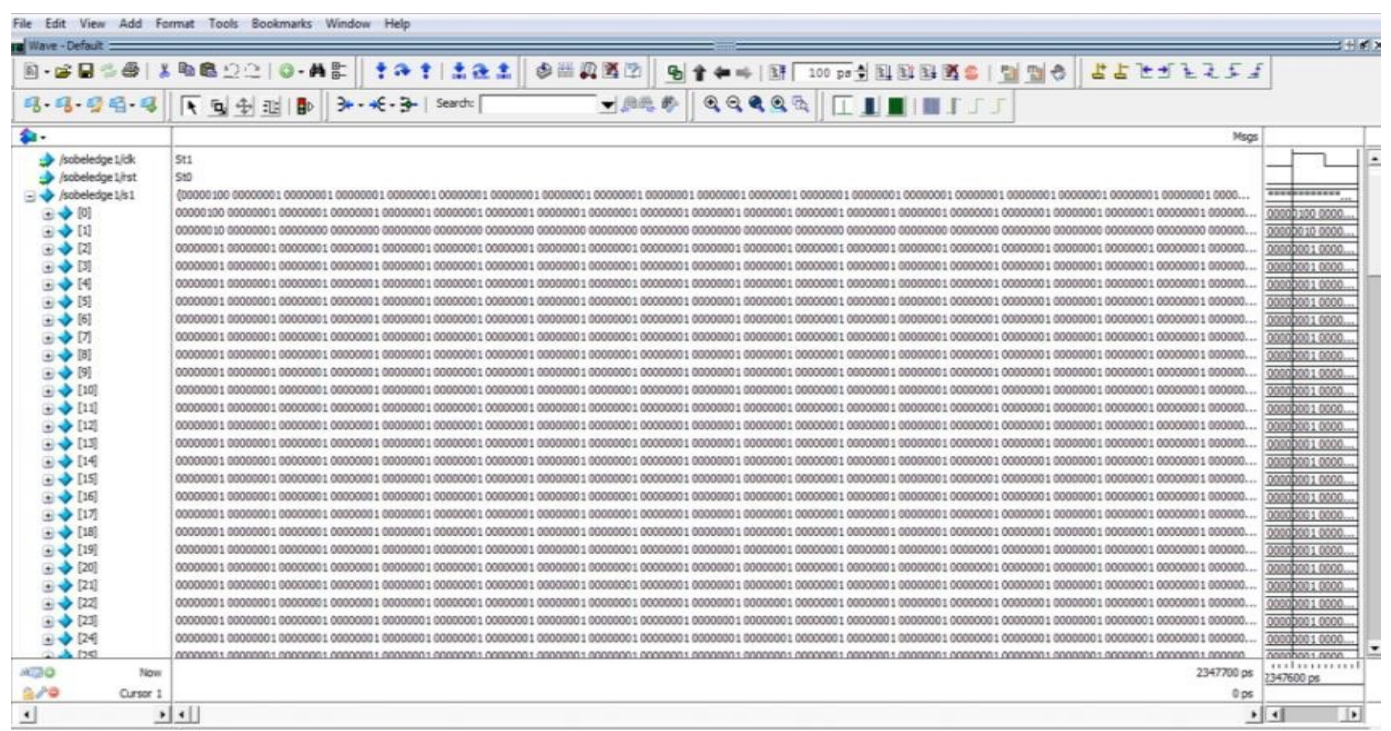

Figure 9. Output waveform of pixel storage in SRAM.

Source: own elaboration.

c) Hardware Implementation Results:

The Hardware implementation includes the implementation of Sobel edge detection algorithm in Spartan-3 Tyro plus FPGA board. The output is seen in a separate virtual image processing window. Figure 10 shows the overall setup for the hardware implementation of edge detection algorithm using FPGA.

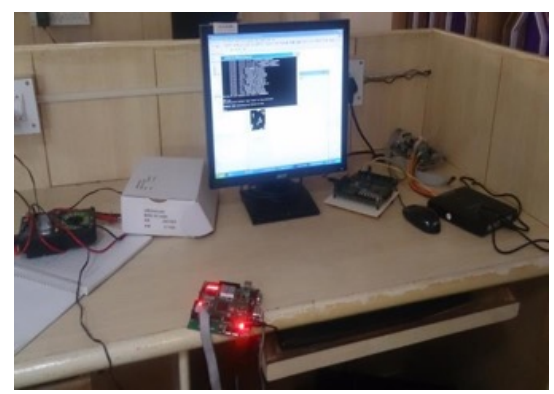

Figure 10. Output Hardware Implementation Setup.

Source: own elaboration. 
d) Results for Sobel Edge Detection Algorithm (Hardware Implementation):

Figure 11 shows the overall output for the Sobel Edge Detection algorithm implemented in FPGA using EDK. Table 1 shows the time constrain for debugging, processor clock frequency and memory constrain for Sobel Edge Detection algorithm.

Table 1. Timing Constraint for Sobel Edge Detection Algorithm (Sample 2).

\begin{tabular}{|r|r|r|}
\hline & Post Synthesis Clock Limits & \\
\hline MODULE & CLOCK PORT & MAX FREQUENCY \\
\hline debug_module & debug_module/update & $72.495 \mathrm{MHz}$ \\
\hline debug_module & SPLB_Clk & $72.495 \mathrm{MHz}$ \\
\hline debug_module & debug_module/drck_i & $72.495 \mathrm{MHz}$ \\
\hline microblaze_0 & DCACHE_FSL_OUT_CLK & $81.820 \mathrm{MHz}$ \\
\hline microblaze_0 & DBG_CLK & $81.820 \mathrm{MHz}$ \\
\hline microblaze_0 & DBG_UPDATE & $81.820 \mathrm{MHz}$ \\
\hline SRAM_256x32 & MCH_PLB_CLK & $83.549 \mathrm{MHz}$ \\
\hline
\end{tabular}

Source: own elaboration.

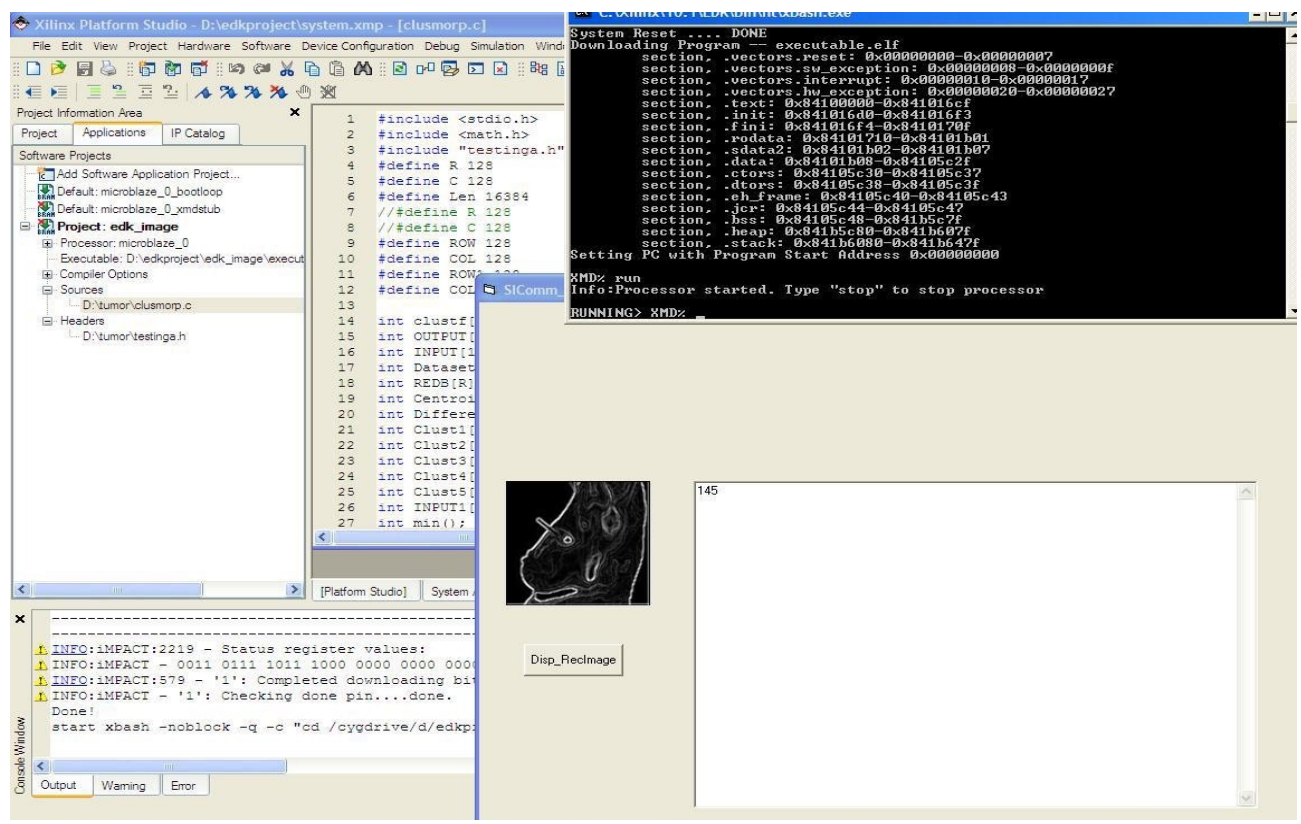

Figure 11. Output for Sobel Edge Detection Algorithm (Sample 2).

Source: own elaboration. 


\section{CONCLUSIONS}

In this work, the clinical decision support system to assist the doctor for diagnosis of breast cancer using thermal image is done. The proposed work has presented the VLSI architecture-based segmentation. In the proposed method, pre-processing has been done by using MATLAB, since the cancer region of the image cannot be easily identified without preprocessing. The Sobel edge detection algorithm is implemented in FPGA using Xilinx Platform Studio in EDK. The timing constraint shows that the Sobel Edge Detection algorithm is more efficient and less time consuming. Image morphing process is to be used to segment the cancer region accurately. The proposed network architecture is modular, compact and efficient and hence it classifies the patients having breast cancer either benign or malignant based on the segmentation using Sobel edge detection algorithm. The outputs obtained are verified with doctors and the obtained outputs are 95\% accurate.

\section{ACKNOWLEDGMENT}

Authors would like to thank their respective management for providing the facilities to carry out this work.

\section{REFERENCES}

American Gancer Society. (2014). Cancer Facts \& Figures 2014. https://www.cancer.org/ research/cancer-facts-statistics/all-cancer-facts-figures/cancer-facts-figures-2014. html

Ankit, A., \& Mandal, M. (2015). Novel hybrid hardware architecture for nuclei detection in skin histopathological images. IEEE International Conference on Signal Processing, Informatics, Communication and Energy Systems (SPICES), 1-6. https://doi.org/10.1109/ SPICES.2015.7091536

Eddy, D. M. (1990). The challenges in Thermal images. Fournal of American Medical Association, 263, 287-290.

Gayathri, R., Madhavi, V., \& Bobby, G. (2015). FPGA Based Diagnosis and Classification of Breast Disease using Thermographic Images. International fournal of scientific research 
and management (IFSRM), 3(6), 3139-3142. https://www.ijsrm.in/index.php/ijssm/ article/view/1100

Hankare, P., Shah, K., Nair, D., \& Nair, D. (2016). Breast cancer detection using thermography. International Research fournal of Engineering and Technology (IRJET), 3(4), 2395-2356. https://www.irjet.net/archives/V3/i4/IRJET-V3I4211.pdf

Kordylewski, H., Graupe, D., \& Liu, K. (2001). A novel large-memory neural network as an aid in medical diagnosis applications. IEEE Transactions on Information Technology in Biomedicine, 5(3), 202- 209. https://ieeexplore.ieee.org/document/945291

Kukar, M.(2003). Transductive reliability estimation in medical diagnosis. Artificial Intelligence in Medicine, 29(1-2), 81-106. https://doi.org/10.1016/S0933-3657(03)00043-5

Kwak, N., \& Choi, G.-H. (2002). Input feature selection for classification problems. IEEE Transactions on Neural Networks, 13(1), 143-159. https://ieeexplore.ieee.org/ document/977291

Li, G., Huang, R., Ding, Z., Gatenby, J. G., Metaxas, D. N., \& Gore, J. G. (2011). A Level Set Method for Image Segmentation in the Presence of Intensity In homogeneities with application to MRI. IEEE Transactions on Image Processing, 20(7), 2007-2016. https:/ /ieeexplore.ieee.org/document/5754584

Lilford, R. J., Pauker, S. G., Braunholtz, D. A., \& Chard, J. (1998). Decision analysis and the implementation of research findings. BMF, 317(7155), 405-409. https://doi. org/10.1136/bmj.317.7155.405

Milosevic, M., Jankovic, D., \& Peulic, A. (2014). Thermography based breast cancer detection using texture features and minimum variance quantization. EXCLI fournal, 13(1), 1204-1215. https://www.ncbi.nlm.nih.gov/pmc/articles/PMC4464488/

Nausheen, N., Seal, A., Khanna, P., \& Halder, S. (2018). A FPGA based implementation of Sobel edge detection. Microprocessors $\mathcal{E}$ Microsystem, 56(1), 84-91. https://doi. org/10.1016/j.micpro.2017.10.011 
Niwas, S. I., Palanisamy, P., Sujathan, K., \& Bengtsson, E. (2013). Analysis of nuclei textures of fine needle aspirated cytology images for breast cancer diagnosis using Complex Daubechies wavelets. Signal Processing, 93(10), 2828-2837. https://doi. org/10.1016/j.sigpro.2012.06.029

Rodrigues, É. O., Gonci, A., Borchartt, T. B., Paiva, A. G., Silva, A. G., \& MacHenry, T. (2014). Comparing results of thermographic images based diagnosis for breast diseases. In IEEE 21st International conference on Systems, Signals and Image Processing (IWSSIP) Proceedings, 39-42. https://ieeexplore.ieee.org/document/6837625

Übeyli, E. D., \& Güler, İ. (2005a). Improving medical diagnostic accuracy of ultrasound Doppler signals by combining neural network models. Computers in biology and medicine, 35(6), 533-554. https://doi.org/10.1016/j.compbiomed.2004.03.006

Übeyli, E. D., \& Güler, İ. (2005b). Feature extraction from Doppler ultrasound signals for automated diagnostic systems. Computers in Biology and Medicine, 35(9), 735-764. https://doi.org/10.1016/j.compbiomed.2004.06.006

XILINX. (n.d.). Spartan 3E starter kit. http://www.xilinx.com/products/boards-and-kits/ hwspar3e-sk-us-g.html 
